\title{
A Phase Noise Analysis Method for Millimeter-Wave Passive Imager BHU-2D-U Frequency Synthesizer
}

\author{
Jin Zhang, Cheng Zheng, Xianxun Yao, and Baohua Yang \\ School of Electronic \& Information Engineering, Beihang University, Beijing, China \\ Correspondence should be addressed to Cheng Zheng; zhengcheng@sina.com
}

Received 3 July 2013; Accepted 13 August 2013

Academic Editors: R. Luzzi and R. Newcomb

Copyright (C) 2013 Jin Zhang et al. This is an open access article distributed under the Creative Commons Attribution License, which permits unrestricted use, distribution, and reproduction in any medium, provided the original work is properly cited.

\begin{abstract}
A nontrivial phase noise analysis method is proposed for frequency synthesizer of a passive millimeter-wave synthetic aperture interferometric radiometer (SAIR) imager for concealed weapon detections on human bodies with high imaging rates. The frequency synthesizer provides local oscillator signals for both millimeter-wave front ends and intermediate frequency IQ demodulators for the SAIR system. The influence of synthesizer phase noise in different offset frequency ranges on the visibility phase errors has been systematically investigated with noise requirements drawn, and the integrated RMS phase error could represent uncorrelated phase noise effects in the most critical offset frequency range for visibility error control. An analytical phase noise simulation method is proposed to guide synthesizer design. To conclude, the phase noise effects on SAIR visibility errors have been concretized to noise design requirements, and good agreements have been observed between simulation and measurement results. The frequency synthesizer designed has been successfully in operation in BHU-2D-U system.
\end{abstract}

\section{Introduction}

Various techniques in the area of concealed weapon detection on human bodies have been developed and widely applied for security checks. Compared with active detection systems, such as the X-ray sensors, the passive SAIR imaging system [1] provides several advantages. It only receives rather than emitting high frequency signals and does not result in human health concerns [2]. Moreover, any concealed hazards, including nonmetallic weapons, can be observed in the obtained image explicitly [3-5].

Visibility error is significant for a SAIR system. Frequency Synthesizer (FS) provides two local oscillators (LO) for $\mathrm{mmW}$ front ends and IF IQ demodulators, and its phase noise (PN) contributes to visibility errors. The PN analysis consists of 3 key problems: determining whether $\mathrm{mmW}$ or IF LO is the major cause of visibility errors from system-level analysis; finding the offset frequency range (OFR) whose $\mathrm{PN}$ is the main contributor of visibility errors and establishes proper SAIR FS phase noise design requirements; finding an accurate PN simulation method to realize FS design and fulfill the requirements.
In Section 2, system configuration and PN system-level modeling are given, and the dominant source of visibility errors from FS is found. In Section 3, this risky PN is separated to three OFRs by visibility error analysis, and SAIR design requirements are proposed. In Section 4, an accurate PN simulation method is given, and FS design is realized. It is found that integrated RMS phase error could represent uncorrelated PN effects that lead to visibility errors. In Section 5, comparisons of simulations and measurements are given. Important conclusions are summarized for FS PN analysis and design of HIR SAIR imager.

\section{PN System-Level Modeling}

A SAIR imager named BHU-2D-U [6] has been developed by Beihang University. It consists of a 48 -element U-shaped antenna array, and each receiver channel [7] is composed of a $\mathrm{mmW}$ receiver [8] and an IF IQ demodulator. The DSP subsystem computes complex cross-correlations between IF IQ output pairs of all receivers simultaneously, with the results calibrated to form visibility samples, whose IFT 


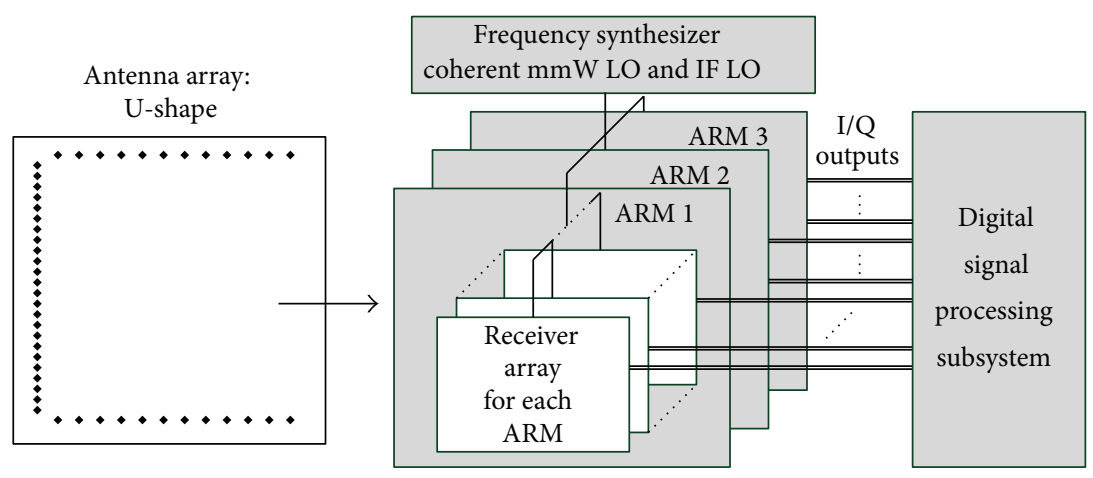

FIGURE 1: System configuration of BHU-2D-U.

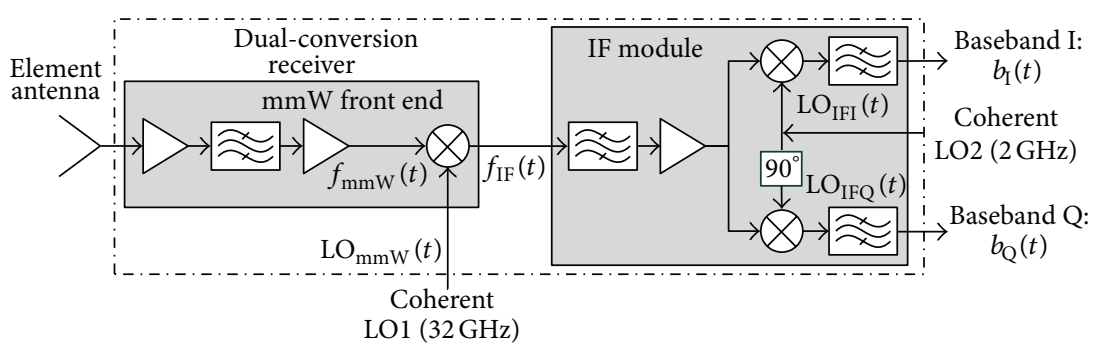

FIgURE 2: Dual-conversion receiver block diagram.

TABLE 1: BHU-2D-U general specifications.

\begin{tabular}{lc}
\hline Parameter & Specification \\
\hline Center frequency & $34 \mathrm{GHz}$ \\
Geometry of antenna array & U Shape \\
Field of view & $20^{\circ}$ (Horizontal) \\
Temperature sensitivity & $36^{\circ}$ (Vertical) \\
Effective distance & $1 \sim 3 \mathrm{~K}$ \\
Range resolution & $2.5-5 \mathrm{~m}$ \\
System bandwidth & $6.5 \mathrm{~cm} \mathrm{at} 3 \mathrm{~m}$ \\
\hline
\end{tabular}

generate the brightness temperature of the field of view. Key parameters of BHU-2D-U are summarized in Table 1, and its simplified block diagram is shown in Figure 1 [6].

A $32 \mathrm{GHz}$ mmW LO and a $2 \mathrm{GHz}$ IF demodulator LO are required for $\mathrm{BHU}-2 \mathrm{D}-\mathrm{U}$. Figure 2 gives the double side-band dual-conversion receiver structure [6]. As $\mathrm{Ku}$ and higher frequency multipliers are easy to be integrated in the $\mathrm{mmW}$ front end, $8 \mathrm{GHz}$ output is selected for FS. Both LO come from the same reference TCXO for coherency. Power divider networks are designed at 2 and $4 \mathrm{GHz}$ for lower loss. The preliminary FS design is shown in Figure 3.

Though previous SAIR researches have given that coherent FS PN has negligible effects on visibility, different receiver channels do produce uncorrelated PN [9] which generates visibility errors. Therefore, it is crucial to concretize PN requirements by system-level modeling and find the dominant PN contributor LO.

2.1. System-Level PN Modeling. The aim of system-level $\mathrm{PN}$ modeling is to concretize $\mathrm{PN}$ effects in the visibility errors. Firstly, it is assumed that phase terms of all signals are constant without phase noise effects. Let $f_{\mathrm{MMW}}(t), \mathrm{LO}_{\mathrm{mmW}}(t), f_{\mathrm{IF}}(t), \mathrm{LO}_{\mathrm{IFI}}(t)$, and $\mathrm{LO}_{\mathrm{IFQ}}(t)$ be the narrow-band $\mathrm{mmW}$ received signal and $\mathrm{mmW}$ mixer $\mathrm{LO}$, IF downconverted signal, and IF I/Q demodulator LOs, as shown in Figure 2. They are given as

$$
\begin{gathered}
f_{\mathrm{mmW}}(t)=A_{\mathrm{mmW}} \cos \left(\omega_{\mathrm{mmW}} t+\emptyset_{\mathrm{mmW}}\right), \\
\mathrm{LO}_{\mathrm{mmW}}(t)=A_{\mathrm{LO} 1} \cos \left(\omega_{\mathrm{LO} 1} t+\emptyset_{\mathrm{LO} 1}\right), \\
\mathrm{LO}_{\mathrm{IFI}}(t)=A_{\mathrm{LO} 2} \cos \left(\omega_{\mathrm{LO} 2} t+\emptyset_{\mathrm{LO} 2}\right), \\
\mathrm{LO}_{\mathrm{IFQ}}(t)=A_{\mathrm{LO} 2} \cos \left(\omega_{\mathrm{LO} 2} t+\emptyset_{\mathrm{LO} 2}+90^{\circ}\right),
\end{gathered}
$$

where $A$ and $\emptyset$ are given as the amplitude and phase terms for each signal, and subscripts LO1 and LO2 represent the first and second LO. $f_{\mathrm{IF}}(t)$ and $b_{i}(t)$ are given as

$$
\begin{gathered}
f_{\mathrm{IF}}(t)=K_{1} f_{\mathrm{mmW}}(t) \mathrm{LO}_{\mathrm{mmW}}(t), \\
b_{\mathrm{I}, \mathrm{Q}}(t)=K_{2} f_{\mathrm{IF}}(t) \mathrm{LO}_{\mathrm{IFI}, \mathrm{Q}}(t),
\end{gathered}
$$

where $K_{1,2}$ refers to the conversion loss of the $\mathrm{mmW}$ and IF mixer. As the mmW mixer is single side band and the IF I/Q 


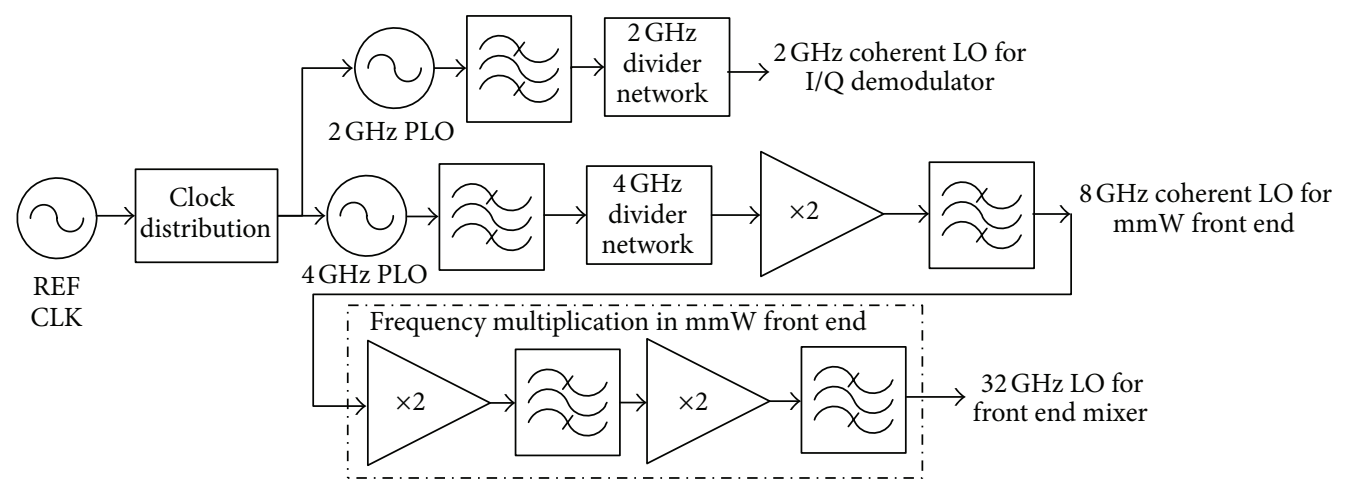

FIGURE 3: Preliminary FS design block diagram.

demodulator is double side band, the phase terms of $b_{\mathrm{I}}(t)$ and $b_{\mathrm{Q}}(t)$ can be given as

$$
\begin{gathered}
\emptyset_{b_{\mathrm{I}}}(t)=\emptyset_{\mathrm{mmW}}-\emptyset_{\mathrm{LO} 1} \pm \emptyset_{\mathrm{LO} 2}, \\
\emptyset_{b_{\mathrm{Q}}}(t)=\emptyset_{\mathrm{mmW}}-\emptyset_{\mathrm{LO} 1} \pm\left(\emptyset_{\mathrm{LO} 2}+90^{\circ}\right) .
\end{gathered}
$$

From (3), the phase terms of IF I/Q demodulator output signals are constants. However, $\mathrm{PN}$ is always present in a realizable $\mathrm{LO}$, whose phase term can be given by

$$
\emptyset_{\mathrm{LO}}(t)=\emptyset_{\mathrm{LO}}+\emptyset_{n}(t) \text {, }
$$

where $\emptyset_{\mathrm{LO}}(t)$ is the phase, $\emptyset_{\mathrm{LO}}$ is the definitive phase, and $\emptyset_{n}(t)$ is the time-variant phase drift caused by PN. In SAIR signal processing, the visibility function sample $V_{i j}$ is obtained by cross-correlations between any I/Q output signals calculated in the UV domain (antenna separation by wavelength), and it is proportional to the expectation of the cross-correlation of $b_{i}(t)$ and $b_{j}(t)$ ( $i$ and $j$ refer to any I/Q demodulated signal, and $i$ can be equal to $j)[1]$ :

$$
\begin{aligned}
V_{i j} & =E\left[b_{i}(t) b_{j}^{*}(t)\right]=E\left[A_{b_{i}(t)} A_{b_{j}(t)}^{*}\right] E\left[e^{j\left(\emptyset_{b_{i}}(t)-\emptyset_{b_{j}}(t)\right)}\right] \\
& =E\left[A_{b_{i}(t)} A_{b_{j}(t)}^{*}\right] E\left[e^{j\left(\mathrm{CPM}_{\mathrm{II}, \mathrm{IQ}}(t)\right)}\right]
\end{aligned}
$$

where $E[\cdot]$ is the expectation operator and ${ }^{*}$ denotes conjugated signal; $A_{b_{i, j}(t)}$ and $\emptyset_{b_{i}}(t)$ are the amplitude and phase of baseband output signal pairs. A new parameter called channel phase mismatch (CPM) is defined for I-I and I-Q cases in (6) as (the same for QQ and QI):

$$
\begin{gathered}
\mathrm{CPM}_{\mathrm{II}}(t)=\Delta \emptyset_{\mathrm{mmW}}+\Delta \emptyset_{\mathrm{LO} 1} \pm \Delta \emptyset_{\mathrm{LO} 2}, \\
\mathrm{CPM}_{\mathrm{IQ}}(t)=\Delta \emptyset_{\mathrm{mmW}}+\Delta \emptyset_{\mathrm{LO} 1} \pm\left(\Delta \emptyset_{\mathrm{LO} 2}-90^{\circ}\right),
\end{gathered}
$$

where $\Delta$ refers to the phase difference between channel pairs. $\mathrm{CPM}$ is thus the combination of phase difference of $\mathrm{mmW}$ received signal $\Delta \emptyset_{\mathrm{mmW}}, \mathrm{mmW}$ mixer LO $\Delta \emptyset_{\mathrm{LO} 1}$ and IF IQ demodulator LO phase mismatches $\Delta \emptyset_{\mathrm{LO} 2}$, and $\Delta \emptyset_{\mathrm{mmW}}$ can be calibrated by point source method. $\Delta \emptyset_{\mathrm{LO} 1}$, and $\Delta \emptyset_{\mathrm{LO} 2}$ could be replaced by (4) as phase mismatches:

$$
\Delta \emptyset_{\mathrm{LO} 1,2}(t)=\Delta \emptyset_{\mathrm{LO} 1,2}+\Delta \emptyset_{n 1,2}(t),
$$

where $\Delta \emptyset_{\mathrm{LO}}$ is definitive and can also be calibrated, but $\Delta \emptyset_{n}(t)$ cannot. Correlated $\mathrm{PN}$ is $\Delta \emptyset_{n}(t)=0$, whereas uncorrelated $\mathrm{PN}$ is $\Delta \emptyset_{n}(t) \neq 0$. The visibility phase error of (5) could be simplified by calibration as:

$$
\begin{aligned}
E\left[e^{j\left(\mathrm{CPM}_{\mathrm{II}, \mathrm{IQ}}(t)\right)}\right] & =E\left[e^{j\left(\Delta \emptyset_{\mathrm{mmW}}+\Delta \emptyset_{\mathrm{LO} 1} \pm \Delta \emptyset_{\mathrm{LO} 2}\right)}\right] \\
& \stackrel{\text { calibration }}{\longrightarrow} E\left[e^{j\left(\Delta \emptyset_{n 1}(t) \pm \Delta \emptyset_{n 2}(t)\right)}\right],
\end{aligned}
$$

where $\Delta \emptyset_{n 1,2}(t)$ represents the uncorrelated $\mathrm{PN}$ in $\mathrm{mmW}$ LOs and IF I/Q demodulator LOs, respectively. In the imaging calculation, phase error expectation of (8) is integrated in time domain and produces error in the imaging results. This integration is given as

$$
E\left[e^{j\left(\Delta \emptyset_{n 1}(t)+\Delta \emptyset_{n 2}(t)\right)}\right]=\frac{1}{\tau_{s}} \int_{0}^{\tau_{s}} e^{j\left(\Delta \emptyset_{n 1}(t)+\Delta \emptyset_{n 2}(t)\right)} \mathrm{dt},
$$

where $\tau_{s}$ is the single image integration time (SIIT). It is clear that $\tau_{s}$ is significant for phase noise analysis and is treated later.

2.2. Dominant Uncorrelated PN LO. Based on the analysis above, it is necessary to determine whether $\Delta \emptyset_{\mathrm{LO} 1}$ or $\Delta \emptyset_{\mathrm{LO} 2}$ is the dominant uncorrelated PN source. As in Figure 3, compared with the IF LO which is generated by the $2 \mathrm{GHz}$ PLO, the mmW LO is multiplied from $4 \mathrm{GHz}$ once to $8 \mathrm{GHz}$ and then twice in the $\mathrm{mmW}$ front ends to $32 \mathrm{GHz}$; hence, the number of components for $\mathrm{mmW}$ mixer LO is greater than for IF LO. Hence the uncorrelated $\mathrm{PN}$ in each $\mathrm{mmW}$ mixer LO is higher than for IF LO, and mmW LO is the dominant uncorrelated PN source.

\section{PN OFR Separation and Requirement Analysis}

As mmW LO is the dominant uncorrelated PN source and PN simulation and analysis are mostly performed in frequency domain, a suitable OFR guideline for $\mathrm{PN}$ analysis is required to define PN requirements over different OFRs. Synthesizer output PN could be further represented by

$$
\emptyset_{n}(t)=A_{n} \cos \left(\omega_{m} t+\psi_{n}\right),
$$


where $\mathrm{PN}$ is given by random amplitude $A_{n}$, offset angular frequency $\omega_{m}=2 \pi f_{m}$, and random phase term $\psi_{n}$. Therefore, the OFRs should be defined by $\tau_{s}$ and $f_{m}$.

3.1. OFR Separation and FS PN Requirements. The phase error expectation is performed in the time domain from 0 to $\tau_{\mathrm{s}}$, but $\mathrm{PN}$ is usually analyzed in the frequency domain. By Fourier Transform, the OFR separations are given by relations between correlation frequency $f_{s}=1 / \tau_{s}$ and PN modulation frequency $f_{m}$ (offset frequency from carrier). A PN separation guideline is proposed for the three OFR separations.

(1) $f_{m} \gg f_{s}$. This range could be interpreted as $f_{m}>100 f_{\mathrm{s}}$ (far offset frequency range (FOFR)). Offset frequency $f_{m}$ is far from the actual correlation region. Phase errors caused by uncorrelated PN in this range are negligible.

(2) $f_{m}<f_{s}$ (very near offset frequency range (VNOFR)). The correlations of visibility functions actually take place in this region, and uncorrelated PN could cause huge visibility errors. By (9), it is necessary to give limits on phase mismatch and temperature drifts instead of PN:

$$
\begin{gathered}
\Delta \phi_{\mathrm{LO} i}-\Delta \phi_{\mathrm{OL} j}<1 \mathrm{deg}, \\
\Delta \phi_{\mathrm{LO} i}-\Delta \phi_{\mathrm{OL} j}<0.5 \mathrm{deg} /{ }^{\circ} \mathrm{C},
\end{gathered}
$$

where $\Delta \phi_{\mathrm{LO} i, j}$ refers to the phase mismatch between different receiver pairs for the same LO.

(3) $f_{s}<f_{m}<100 f_{s}$. This range is named as middle offset frequency range (MOFR). The $\mathrm{PN}$ variations in this region are apparent, and they degrade phase matches in VNOFR by uncorrelated PN between receiver pairs. Therefore, stringent PN simulations and phase error simulations are required. As PN changes rapidly, integrated RMS phase error [10-12] is applied to limit PN for both 4 and $8 \mathrm{GHz}$ LOs in this range:

$$
\begin{gathered}
\operatorname{PhEr}_{\mathrm{RMS}, 4 \mathrm{GHz}}=\sqrt{2 \times \int_{f_{s}}^{100 f_{s}} \mathrm{PN}(f) \mathrm{df}}<1.3 \mathrm{deg}, \\
\mathrm{PhEr}_{\mathrm{RMS}, 8 \mathrm{GHz}}=\sqrt{2 \times \int_{f_{s}}^{100 f_{s}} \mathrm{PN}(f) \mathrm{df}}<2.4 \mathrm{deg},
\end{gathered}
$$

where the coefficient 2 refers to double side-band PN integration, and this threshold is more stringent than MIRAS (1 deg for single side-band LO) [9]. Now $\tau_{s}$ is required to concretize OFR separations.

3.2. $\tau_{s}$ (SIIT) Derivation by Security Imaging $\Delta T$. As HIR is required by security imaging, video rate imaging with very short SIIT is preferred, but SIIT is limited by the least temperature sensitivity required for effective hazard identification, which is given by [1]

$$
\Delta T \approx \frac{1}{\eta} \frac{T_{A}+T_{R}}{\sqrt{B \tau_{s t} / 2.46}} \frac{\alpha_{W}}{\alpha_{F}} \alpha_{\mathrm{LO}} \sqrt{N_{V}},
$$

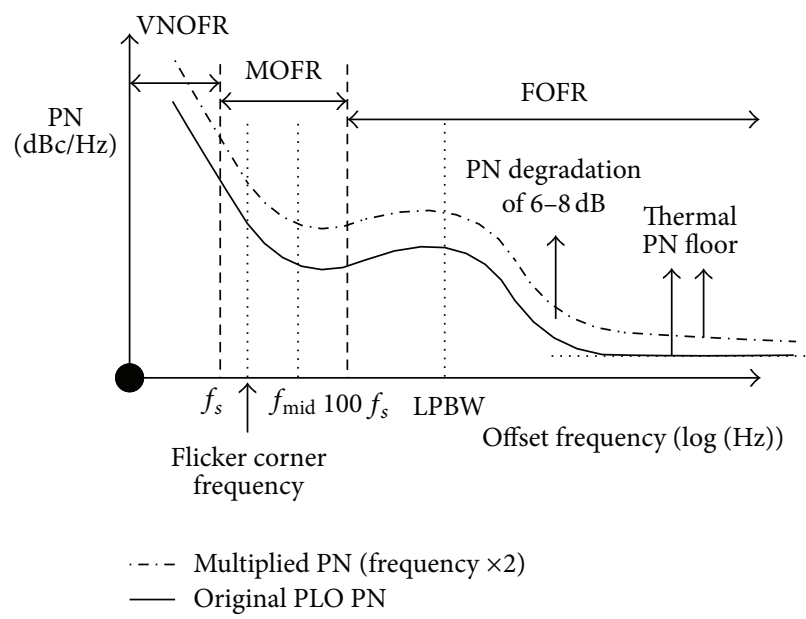

FIgURE 4: OFR separation for FS PN analysis (closed-loop PLO).

where $\eta$ is element antenna efficiency ( 0.65 of Bode horn); $T_{A}(300 \mathrm{~K})$ and $T_{R}(400 \mathrm{~K})$ are antenna and receiver noise temperatures; $\alpha_{W}$ is the window factor ( 0.53 for hamming window); $\alpha_{F}$ is the filter factor (1.19 for gauss prediction); $N_{V}$ is the sampling point number for rectangular visibility function $(49 \times 25=1225) ; B$ is system bandwidth of $200 \mathrm{MHz}$ [13]. The least brightness temperature discrepancy for hazards and human body is $3 \mathrm{~K}$, in which $\Delta T$ must be less than [6].

The total integration time $\left(\tau_{s t}\right)$ is related to the dynamic high imaging rate (HIR) application condition of security imaging. If a person goes through a $2.5 \mathrm{~m}$ effective imaging distance with an average walking speed of $1.4 \mathrm{~m} / \mathrm{s}$, the dwell time is about $1.8 \mathrm{~s} . \tau_{s t}$ should be within $1 \mathrm{~s}$ for 10 images $(0.1 \mathrm{~s}$ for each image). $0.5 \mathrm{~s} \tau_{s t}$ corresponds to a sensitivity of $2.63 \mathrm{~K}$, which leaves some margin from $3 \mathrm{~K}$ limit. Thus, the $0.05 \mathrm{~s} \tau_{s}$ (minimum SIIT) is used for OFR separation.

\section{PN Simulation Method}

Based on the analysis above, a typical OFR separation is shown in Figure 4. It is important to simulate PN in each OFR to obtain an accurate PN curve and estimate whether the requirements of (12) are realized. The method is given for a single-loop PLO FS design (the most common and cost-effective method), and PN simulation for frequency multiplication is also proposed.

Two important parameters in single-loop PLO PN simulation are the flicker corner frequency $\left(f_{\text {flicker }}\right)$ and loop bandwidth (LPBW). $f_{\text {flicker }}$ is the flicker noise transition frequency of the reference oscillator and determined by its output stage component. A high-Q and low flicker corner, low PN silicon bipolar TCXO is preferred $[14,15]$ (our TCXO flicker corner is $80 \mathrm{~Hz}$ [16]). LPBW controls the dynamic and noise traits of the loop as well as spur attenuation and is usually set from tens to hundreds of $\mathrm{kHz}$.

4.1. $4 \mathrm{GHz}$ LO PN Simulation for Each OFR. Conventional time-consuming PLO PN simulation method $[17,18]$ is performed by closed-loop transfer function with detailed 
loop filter design and $\mathrm{PN}$ data from each contributor (TCXO, Phase Frequency Detector (PFD), VCO, and Divider). This method is designed for a quick and accurate simulation of $\mathrm{PN}$ specifically for the separated OFRs of BHU-2D-U FS.

(1) FOFR PN $\left(f_{m}>2 \mathrm{kHz}\right)$. Part of this range is inside LPBW, and the other is outside. PN in the in-LPBW part is mainly caused by reference, PLO chip and frequency divider, while in the other part VCO is the main noise contributor [14].

(2) VNOFR PN $\left(f_{m}<20 \mathrm{~Hz}\right)$. PN in this very near range is caused by reference oscillator noise degraded by $20 \lg (N) ; N=f_{\mathrm{RF}} / f_{\mathrm{PFD}}$ is the multiplier coefficient for integer PLO' For $20 \mathrm{~Hz}$ is lower than reference oscillator $f_{\text {flicker }}$.

(3) MOFR PN. This region covers both the multiplied reference noise region and the in-LPBW PFD noise region. $\mathrm{PN}$ simulation can be further divided into two regions.

(a) $20 \mathrm{~Hz}<f_{m} \leq f_{\text {flicker }}=80 \mathrm{~Hz}$. PN in this region is mostly dominated by the degraded reference PN. Reference PN can be given by the modified Leeson Model $[19,20]$ as

$$
\mathrm{PN}_{\text {ref }}(\text { Linear })=\mathrm{PN}_{\text {floor }}\left(1+\frac{f_{c}^{2}}{f_{m}^{2}}\right)\left(1+\frac{f_{\text {flicker }}}{f_{m}}\right) \text {, }
$$

where $\mathrm{PN}_{\text {floor }}$ is the $\mathrm{PN}$ floor and $f_{c}$ is the corner frequency $(20 \mathrm{~dB} / \mathrm{dec}$ to flat noise floor) [16]. The PN can be given as

$$
\mathrm{PN}_{\mathrm{PLO}}(\mathrm{dBc} / \mathrm{Hz})=\mathrm{PN}_{\text {ref }}+20 \lg (N)+\mathrm{PN}_{\mathrm{deg}} \text {, }
$$

where $\mathrm{PN}_{\mathrm{deg}}$ is a degradation of 3-4 $\mathrm{dB}$ by other noise sources. A lower $N$ and a low $\mathrm{PN}$ reference resonators are effective to minimize $\mathrm{PN}$ [21]. VCO PN in this region is usually suppressed by $30 \sim 40 \mathrm{~dB}$; thus, it is not considered.

(b) $80 \mathrm{~Hz}<f_{m}<2 \mathrm{kHz}$. In this range, PFD, frequency divider, and other noises come in, the multiplied reference noise from (13) is degraded, and the noise slope becomes smaller. Conventional in-LPBW PN simulation method assumes $\mathrm{PN}$ remains unchanged from $f_{\text {flicker }}$ to LPBW as

$$
\mathrm{PN}_{\mathrm{PLO}}(\mathrm{dBc} / \mathrm{Hz})=\mathrm{PN}_{\text {floor }}(1 \mathrm{~Hz})+10 \lg \left(f_{\mathrm{PFD}}\right)+20 \lg (N) \text {. }
$$

There are two obvious drawbacks. Firstly, $\mathrm{PN}$ in this region is not constant; secondly, PFD frequency specified in the datasheet cannot be applied in every design. A modified method introduces a new mean frequency $f_{\text {mid }}\left(f_{\text {mid }}=\right.$ $\left.\sqrt{f_{\text {flicker }} \times \text { LPBW }}\right)$, with its PN calculated by

$$
\begin{aligned}
\mathrm{PN}_{f \text { mid }}(\mathrm{dBc} / \mathrm{Hz})= & \mathrm{PN}_{\text {floor }}(1 \mathrm{~Hz})+10 \lg \left(\frac{f_{\mathrm{PFD}}}{f_{\mathrm{PFD}_{-} G}}\right) \\
& +10 \lg \left(f_{\mathrm{PFD}}\right)+20 \lg (N)
\end{aligned}
$$

where $\mathrm{PN}_{\text {floor }}(1 \mathrm{~Hz})$ is the normalized $1 \mathrm{~Hz} \mathrm{PN}$ floor at a specified PFD frequency ( $f_{\text {PFD_G }}$ ) from datasheet, and $f_{\mathrm{PFD}}$ is the actual PFD. A negative slope of about $-13 \mathrm{~dB} / \mathrm{dec}$ is employed between $f_{\text {flicker }}$ and $f_{\text {mid }}$. A noise peaking of $3 \sim 5 \mathrm{~dB}$ is always present between $f_{\text {mid }}$ and LPBW. Therefore, PN can be simulated by $f_{\text {flicker }}$, the negative slope and LPBW.

4.2. Multiplier Output PN Simulation. Once the $4 \mathrm{GHz} \mathrm{PN}$ has been simulated, the $\mathrm{PN}$ of $8 \mathrm{GHz}, 16 \mathrm{GHz}$ and $32 \mathrm{GHz}$ can be proposed by

$$
\mathrm{PN}_{\mathrm{MUL}}(\mathrm{dBc} / \mathrm{Hz})=\mathrm{PN}_{\mathrm{PLO}}+20 \lg \left(\frac{f_{\mathrm{MUL}}}{f_{\mathrm{PLO}}}\right)+\mathrm{PN}_{\mathrm{deg}},
$$

where $\mathrm{PN}_{\mathrm{MUL}}$ is the output $\mathrm{PN}$ of frequency multiplier, and $f_{\text {MUL }}$ and $f_{\text {PLO }}$ represent multiplier output and PLO output frequency, respectively. $\mathrm{PN}_{\mathrm{deg}}$ is the $\mathrm{PN}$ degradation coefficient of $1 \sim 2 \mathrm{~dB}$ due to power noise. The degradation of $\mathrm{PN}$ for each multiplied frequency is $6 \sim 8 \mathrm{~dB}$. The parameters used in the above method are shown in Figure 4 with only upper side-band PN depicted.

\section{Results and Discussions}

5.1. Synthesizer Design. The design of FS PLO is performed with reference to the PN simulation method above. As FOFR $\mathrm{PN}$ is negligible and its main PN contributor is VCO, a PLO chip with an on-chip VCO is preferred, which is lower in cost than multichip designs. From MOFR discussions, large PFD frequency is preferred (also smaller $N$ ); thus, a chip with a larger PFD frequency is preferred [22-24]. Other loop parameters such as $-65 \mathrm{dBc}$ spur control, LPBW, and phase margin are required. A common rule for $\mathrm{LPBW}$ is $1 / 10 \sim 1 / 20$ of PFD frequency [25]. A narrow LPBW trades lock time for steeper attenuation on PN and spurs but may cause inLPBW PN to rise [26]. $40 \mathrm{kHz}$ and $100 \mathrm{kHz}$ are set for LPBWs from MOFR PN control and spur requirements (limited to $-65 \mathrm{dBc}$ for $8 \mathrm{GHz}$ ). The common rule of phase margin is 30 $55 \mathrm{deg}$, and $45 \mathrm{deg}$ is a balanced choice between oscillatory and sluggish loops [27]. A 3rd-order loop filter is designed on known methods [22, 28].

5.2. PN, RMS Phase Error Simulations and Test Results. PN profiles are simulated by the above methods and also measured [29] (Figure 5). Simulation and measurement results are given in Tables 2 and 3 and Figure 6 .

It is clearly observed from Tables 2 and 3 that the PN simulations are in good agreement with test results. $\mathrm{PN}$ in the VNOFR $(10 \mathrm{~Hz})$, MOFR $(100$ and $1 \mathrm{kHz})$, and FOFR $(\geq 10 \mathrm{kHz})$ all agree well with measurement results. From PN measurements in Figure 6, it is observed that PN degradation due to frequency multiplication also agrees well with the simulations.

The integrated RMS phase error simulations in Tables 4 and 5 are obtained from PN simulations in Tables 2 and 3 , and good agreement has been observed with test results. $\mathrm{PHEr}_{\mathrm{RMS}, 4 \mathrm{GHz}}$ and $\mathrm{PHEr}_{\mathrm{RMS}, 8 \mathrm{GHz}}$ are limited to $1.3 \mathrm{deg}$ and 2.4 deg (double sideband), by which BHU-2D FS PN requirements of (12) are reached. 


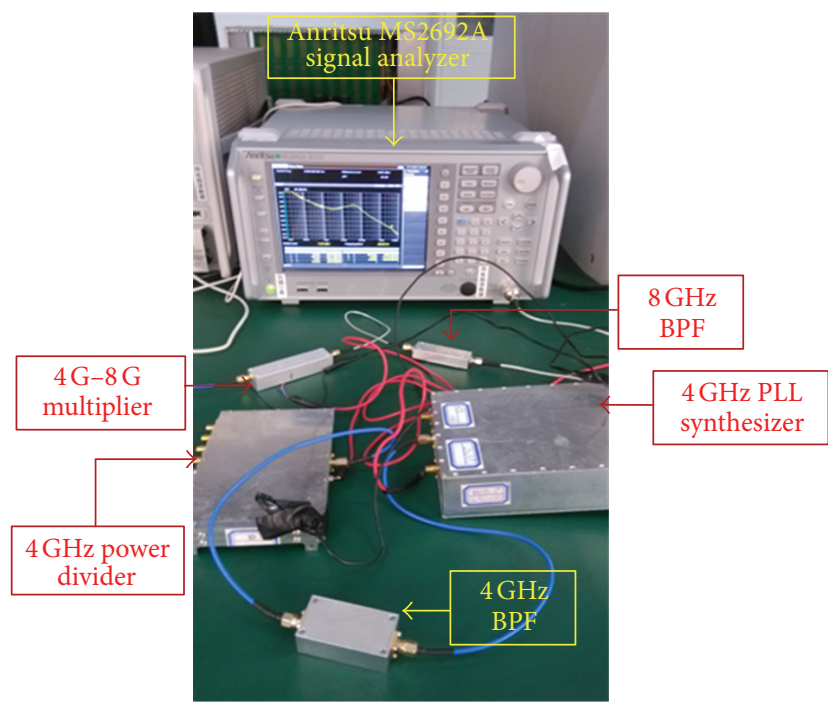

FIGURE 5: $8 \mathrm{GHz}$ phase noise measurement setup.

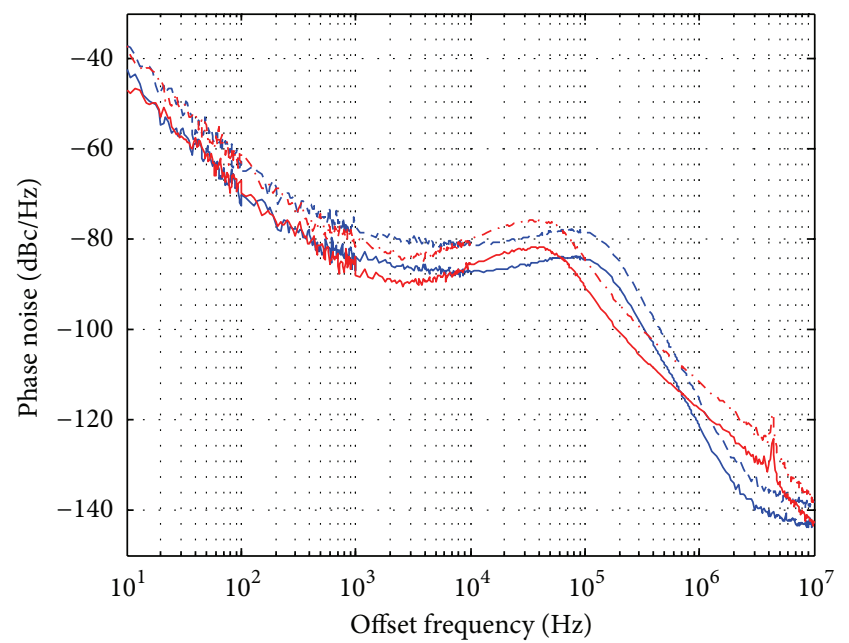

- LPBW $=100 \mathrm{kHz}, 4 \mathrm{GHz} \quad$ LPBW $=40 \mathrm{kHz}, 4 \mathrm{GHz}$ - - - $\mathrm{LPBW}=100 \mathrm{kHz}, 8 \mathrm{GHz} \quad$...- $\mathrm{LPBW}=40 \mathrm{kHz}, 8 \mathrm{GHz}$

FIGURE 6: Phase noise measurement results.

5.3. LPBW Discussions for SAIR FS Design. There are several findings on LPBW from above results. Firstly, as lock time is not specified, LPBW is noncritical for SAIR LOs, and different LPBWs yield similar PN and phase errors in MOFR. In a wider frequency range $(10 \mathrm{~Hz} \sim 10 \mathrm{MHz})$, wide LPBW does lead to more integrated RMS phase error (13\% more, from $6.053-6.842 \mathrm{deg}$ for $8 \mathrm{GHz})$, but narrow $(40 \mathrm{kHz})$ or wide $(100 \mathrm{kHz})$ LPBW PLO yields nearly identical phase error results in MOFR, with only 2.4\% rise from 2.216 deg to $2.268 \mathrm{deg}$ for $8 \mathrm{GHz}$. As a higher LPBW leads to a smoother $\mathrm{PN}$ profile without degradation to design requirements, it is preferred in SAIR FS design.
TABLE 2: Phase noise test results (LPBW $=40 \mathrm{kHz}$ ).

\begin{tabular}{lccc}
\hline Parameter & $\begin{array}{c}\text { Offset Freq. } \\
(\mathrm{Hz})\end{array}$ & $\begin{array}{c}\text { Simulation } \\
(\mathrm{dBc} / \mathrm{Hz})\end{array}$ & $\begin{array}{c}\text { Measurement } \\
(\mathrm{dBc} / \mathrm{Hz})\end{array}$ \\
\hline & 10 & -39.97 & -47.00 \\
& 100 & -69.97 & -69.72 \\
$4 \mathrm{GHz}$ & $1 \mathrm{~K}$ & -85.58 & -87.94 \\
phase noise & $10 \mathrm{~K}$ & -92.58 & -85.69 \\
& $100 \mathrm{~K}$ & -95.45 & -90.75 \\
& $1 \mathrm{M}$ & -120.45 & -117.56 \\
& $10 \mathrm{M}$ & -146.45 & -142.69 \\
\hline & 10 & -33.98 & -37.07 \\
phase noise & 100 & -63.98 & -61.02 \\
& $1 \mathrm{~K}$ & -79.58 & -80.65 \\
& $10 \mathrm{~K}$ & -76.58 & -80.16 \\
& $100 \mathrm{~K}$ & -83.45 & -84.87 \\
& $1 \mathrm{M}$ & -112.45 & -111.54 \\
& $10 \mathrm{M}$ & -137.45 & -137.68 \\
\hline
\end{tabular}

TABLE 3: Phase noise test results (LPBW $=100 \mathrm{kHz})$.

\begin{tabular}{lccc}
\hline Parameter & $\begin{array}{c}\text { Offset Freq. } \\
(\mathrm{Hz})\end{array}$ & $\begin{array}{c}\text { Simulation } \\
(\mathrm{dBc} / \mathrm{Hz})\end{array}$ & $\begin{array}{c}\text { Measurement } \\
(\mathrm{dBc} / \mathrm{Hz})\end{array}$ \\
\hline & 10 & -39.97 & -42.58 \\
& 100 & -69.97 & -68.66 \\
$4 \mathrm{GHz}$ & $1 \mathrm{~K}$ & -85.79 & -86.77 \\
phase noise & $10 \mathrm{~K}$ & -95.98 & -94.77 \\
& $100 \mathrm{~K}$ & -90.98 & -90.36 \\
& $1 \mathrm{M}$ & -120.45 & -117.30 \\
& $10 \mathrm{M}$ & -146.45 & -142.44 \\
\hline & 10 & -33.97 & -37.96 \\
phase noise & 100 & -63.97 & -62.79 \\
& $1 \mathrm{~K}$ & -79.79 & -80.94 \\
& $10 \mathrm{~K}$ & -89.98 & -88.91 \\
& $100 \mathrm{~K}$ & -83.98 & -84.40 \\
& $1 \mathrm{M}$ & -112.45 & -111.32 \\
& $10 \mathrm{M}$ & -137.45 & -137.39 \\
\hline
\end{tabular}

TABLE 4: Integrated RMS phase RMS results (LPBW $=40 \mathrm{kHz})$.

\begin{tabular}{lccc}
\hline Parameter & Offset Freq. (Hz) & Simulation & Measurement \\
\hline \multirow{3}{*}{ PHEr $_{\mathrm{RMS}, 4 \mathrm{GHz}}$} & $20 \sim 80$ & $1.098 \mathrm{deg}$ & $1.064 \mathrm{deg}$ \\
& $80 \sim 2 \mathrm{~K}$ & $0.448 \mathrm{deg}$ & $0.455 \mathrm{deg}$ \\
& $20 \sim 2 \mathrm{~K}$ & $1.170 \mathrm{deg}$ & $1.158 \mathrm{deg}$ \\
& $10 \sim 10 \mathrm{M}$ & $2.875 \mathrm{deg}$ & $3.082 \mathrm{deg}$ \\
\hline $\mathrm{PHEr}_{\mathrm{RMS}, 8 \mathrm{GHz}}$ & $20 \sim 80$ & $2.015 \mathrm{deg}$ & $1.984 \mathrm{deg}$ \\
& $80 \sim 2 \mathrm{~K}$ & $1.007 \mathrm{deg}$ & $0.989 \mathrm{deg}$ \\
& $20 \sim 2 \mathrm{~K}$ & $2.275 \mathrm{deg}$ & $2.216 \mathrm{deg}$ \\
& $10 \sim 10 \mathrm{M}$ & $6.064 \mathrm{deg}$ & $6.053 \mathrm{deg}$ \\
\hline
\end{tabular}


TABLE 5: Integrated RMS phase RMS results $(\mathrm{LPBW}=100 \mathrm{kHz})$.

\begin{tabular}{lccc}
\hline Parameter & Offset Freq. (Hz) & Simulation & Measurement \\
\hline \multirow{2}{*}{ PHEr $_{\mathrm{RMS}, 4 \mathrm{GHz}}$} & $20 \sim 80$ & $0.942 \mathrm{deg}$ & $0.957 \mathrm{deg}$ \\
& $80 \sim 2 \mathrm{~K}$ & $0.501 \mathrm{deg}$ & $0.515 \mathrm{deg}$ \\
& $20 \sim 2 \mathrm{~K}$ & $1.114 \mathrm{deg}$ & $1.086 \mathrm{deg}$ \\
& $10 \sim 10 \mathrm{M}$ & $3.351 \mathrm{deg}$ & $3.469 \mathrm{deg}$ \\
\hline $\mathrm{PHEr}_{\mathrm{RMS}, 8 \mathrm{GHz}}$ & $20 \sim 80$ & $2.179 \mathrm{deg}$ & $2.039 \mathrm{deg}$ \\
& $80 \sim 2 \mathrm{~K}$ & $1.032 \mathrm{deg}$ & $1.049 \mathrm{deg}$ \\
& $20 \sim 2 \mathrm{~K}$ & $2.302 \mathrm{deg}$ & $2.268 \mathrm{deg}$ \\
& $10 \sim 10 \mathrm{M}$ & $6.815 \mathrm{deg}$ & $6.842 \mathrm{deg}$ \\
\hline
\end{tabular}

TABLE 6: MIRAS and BHU-2D-U OFR comparison.

\begin{tabular}{lcc}
\hline Parameter & MIRAS & BHU-2D-U \\
\hline Integration time & $1.2 \mathrm{~s}$ & $0.05 \mathrm{~s}$ \\
FOFR & $>100 \mathrm{~Hz}$ & $>2 \mathrm{kHz}$ \\
VNOFR & $<1 \mathrm{~Hz}$ & $<20 \mathrm{~Hz}$ \\
MOFR & $1 \mathrm{~Hz} \sim 100 \mathrm{~Hz}$ & $20 \mathrm{~Hz} \sim 2 \mathrm{kHz}$ \\
\hline
\end{tabular}

\section{Conclusion}

In this paper, $\mathrm{PN}$ effects on visibility errors for BHU-2D-U SAIR system have been investigated and concretized to synthesizer PN design requirements from system-level analysis. FS design is given accordingly, and good agreements between simulation and measurement results is observed, with the following conclusions drawn.

For PN OFR separations and requirements, the conclusions are as follows. (1) Due to HIR imaging requirement of BHU-2D-U, OFR separation is different from MIRAS, as shown in Table 6. BHU-2D-U MOFR spreads much further into higher offset frequencies than MIRAS. For MIRAS, the MOFR stays within the flicker noise corner of the reference oscillator, but BHU-2D-U MOFR covers a part of in-LPBW $\mathrm{PN}$ that is out of flicker noise region. (2) VNOFR incurred phase error cannot be quantified by PN, and FOFR PN has negligible effects on visibility phase errors. MOFR is the main contributor of uncorrelated PN that can be controlled by FS PN. (3) The PN simulation method is proposed in detail for the newly divided OFRs. The MOFR is further divided by $f_{\text {flicker }}$, and $\mathrm{PN}$ and integrated RMS phase errors are simulated accurately. Measurement results of integrated RMS phase errors are better than those of MIRAS specifications.

From SAIR FS design perspective, the findings are as follows. (1) As lock time is not important, there is plenty of freedom on LPBW. (2) A slightly higher LPBW leads to smoother in-LPBW PN without noticeable degradation for MOFR phase error requirement of (12). (3) As all interested OFRs are inside LPBW, VCO noise contribution is usually not critical. A low-cost on-chip VCO works well for SAIR synthesizer.

The PN analysis method has been proved to fulfill the specific SAIR phase noise requirements, and the synthesizer designed has been successfully in operation in BHU-2D$\mathrm{U}$ system. Future research works include improvements on
$\mathrm{PN}$ and its effects on imaging. These optimizations are also related to system-level improvement plans.

\section{References}

[1] A. Camps, Application of interferometric radiometry to earth observation [Ph.D. thesis], Polytechnic University of Catalonia, Barcelona, Spain, 1996.

[2] T. D. Williams and N. M. Vaidya, "A compact, low-cost, passive MMW security scanner," in Proceedings of the Passive Millimeter-Wave Imaging Technology VIII, pp. 109-116, Orlando, Fla, USA, March 2005.

[3] D. Nötel, J. Huck, S. Neubert, S. Wirtz, and A. Tessmann, "A compact $\mathrm{mmW}$ imaging radiometer for concealed weapon detection," in Proceedings of the Joint 32nd International Conference on Infrared and Millimetre Waves, and 15th International Conference on Terahertz Electronics (IRMMW-THz '07), pp. 269-270, Cardiff, UK, September 2007.

[4] V. G. Kolinko, S.-H. Lin, A. Shek et al., "A passive millimeterwave imaging system for concealed weapons and explosives detection," in Proceedings of the Optics and Photonics in Global Homeland Security, pp. 85-92, Orlando, Fla, USA, April 2005.

[5] C. A. Martin and V. G. Kolinko, "Concealed weapons detection with an improved passive millimeter-wave imager," in Proceedings of the Radar Sensor Technology VIII and Passive MillimeterWave Imaging Technology VII, pp. 252-259, Orlando, Fla, USA, April 2004.

[6] C. Zheng, X. Yao, A. Hu, and J. Miao, "A passive Millimeterwave imager used for concealed weapon detection," Progress in Electromagnetic Research B, vol. 46, pp. 379-397, 2013.

[7] G. Mehdi, H. Anyong, and M. Jungang, "Millimetre-wave all symmetric edge-coupled bandpass filter," in Proceedings of the 10th International Symposium on Antennas, Propagation \& EM Theory, Xi'an, China, October 2012.

[8] B. H. Yang, G. Mehdi, A. Y. Hu et al., "The round ended design and measurement of all symmetric edge-coupled bandpass filter," Progress in Electromagnetic Research C, vol. 38, pp. 191203, 2013.

[9] F. Torres, I. Corbella, E. Castro, M. Segarraand, and R. Vilaseca, "Phase noise requirements in interferometric radiometers," in Proceedings of the IEEE International Geoscience and Remote Sensing Symposium (IGARSS '09), pp. III1027-III1030, Cape Town, South Africa, July 2009.

[10] B. Brannon, "Sampled systems and the effects of clock phase noise and jitter," Technical Report, Analog Devices Inc, AN-756.

[11] M. Shinagawa, Y. Akazawa, and T. Wakimoto, "Jitter analysis of high-speed sampling systems," IEEE Journal of Solid-State Circuits, vol. 25, no. 1, pp. 220-224, 1990.

[12] W. Kester, "Converting oscillator phase noise to time jitter," Tech. Rep. MT-008, Analog Devices.

[13] C. Zheng, X. Yao, A. Hu et al., "Closed form calibration of 1 bit/2level correlator used for synthetic aperture interferometric radiometer," Progress in Electromagnetic Research M, vol. 29, pp. 193-205, 2013.

[14] A. Chenakin, "Building a microwave frequency synthesizerpart 1: getting started," High Frequency Electronics, pp. 58-65, 2008.

[15] A. Chenakin, "Building a microwave frequency synthesizerPart 2: component selection," High Frequency Electronics, pp. 18-26, 2008. 
[16] 7. $0 \times$ 5. $0 \mathrm{~mm}$ SMD High Precision Voltage Controlled Temperature Compensated Crystal Oscillator, TAITIEN Electronics Co. Ltd.

[17] L. Lascari, "Accurate phase noise prediction in PLL synthesizers: here is a method that uses more complete modeling for wireless applications," Applied Microwave and Wireless, vol. 12, no. 4, pp. 30-38, 2000.

[18] L. Lascari, "Accurate phase noise prediction in PLL synthesizers. Part 2: here is a method that uses more complete modeling for wireless applications," Applied Microwave and Wireless, vol. 12, no. 5, pp. 90-96, 2000.

[19] D. B. Leeson, "Simple model of feedback oscillator noise spectrum," Proceedings of the IEEE, vol. 54, no. 2, pp. 329-330, 1966.

[20] G. Sauvage, "Phase noise in oscillators: a mathematical analysis of leeson's model," IEEE Transactions on Instrumentation and Measurement, vol. 26, no. 4, pp. 408-410, 1977.

[21] F. L. Dacus, J. Van Niekerk, and S. Bible, “Tracking phase noise in short-range radios," Microwaves and RF, vol. 41, no. 3, pp. 5764, 2002.

[22] D. Banerjee, PLL Performance, Simulation and Design, Texas Instrument, 4th edition, 2006.

[23] C. Barrett, "Frational/Integer-N PLL Basics," Tech. Rep., Texas Instrument, 1999.

[24] D. Banerjee, "Frequency Synthesis and Planning for PLL Architectures," Tech. Rep. AN-1865, Texas Instrument, 2009.

[25] A. Fox, "Ask the application engineer-30, PLL synthesizers," Tech. Rep., Analog Devices, 2002.

[26] R. E. Best, Phase-Locked Loops Design, Simulation and Applications, McGraw-Hill Companies, 5th edition, 2003.

[27] F. Y. Gardner, Phaselock Techniques, John Wiley and Sons, New York, NY, USA, 3rd edition, 2005.

[28] AN-1001, "An analysis and performance evaluation of a passive filter design technique for charge pump PLL's, Tech. Rep., Texas Instrument, 2004.

[29] Signal Analyzers MS2692A Introduction, Technical Documentation, Anritsu Corporation. 

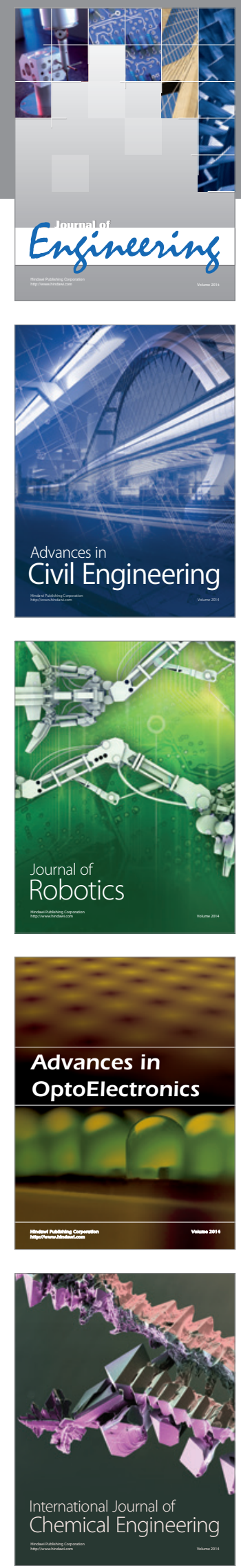

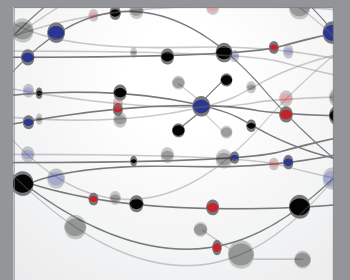

The Scientific World Journal
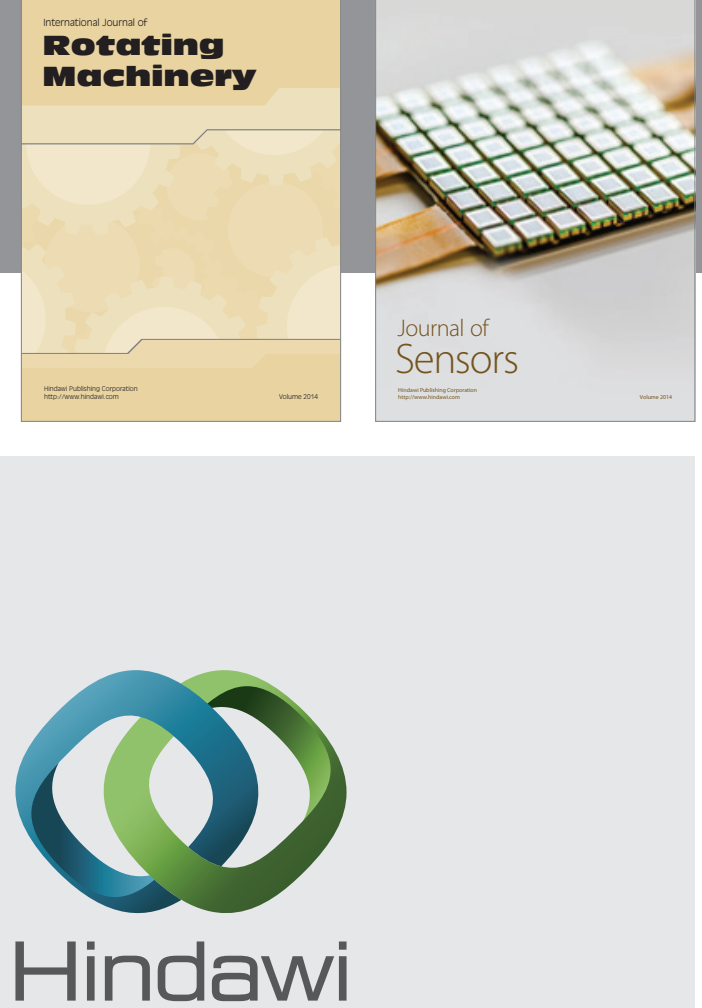

Submit your manuscripts at http://www.hindawi.com
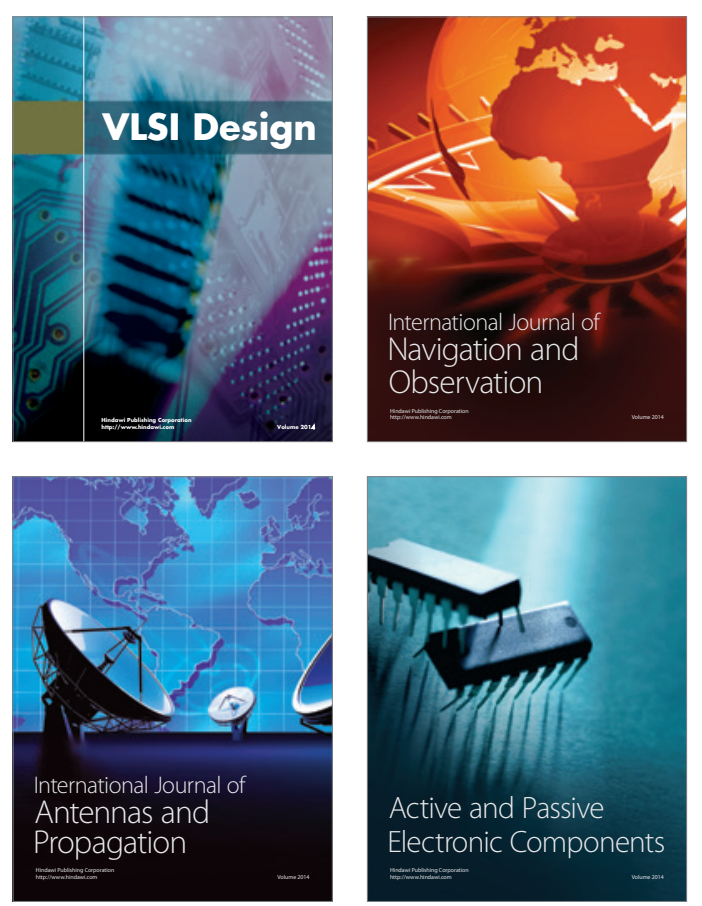
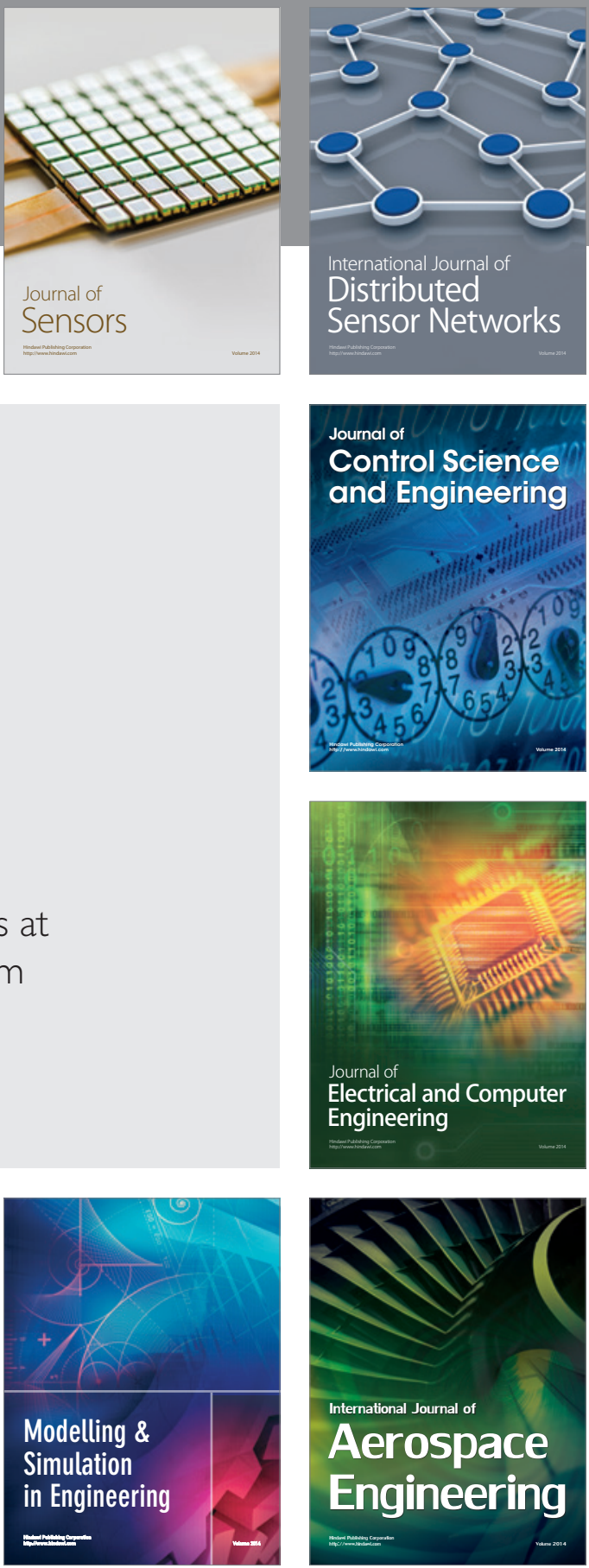

Journal of

Control Science

and Engineering
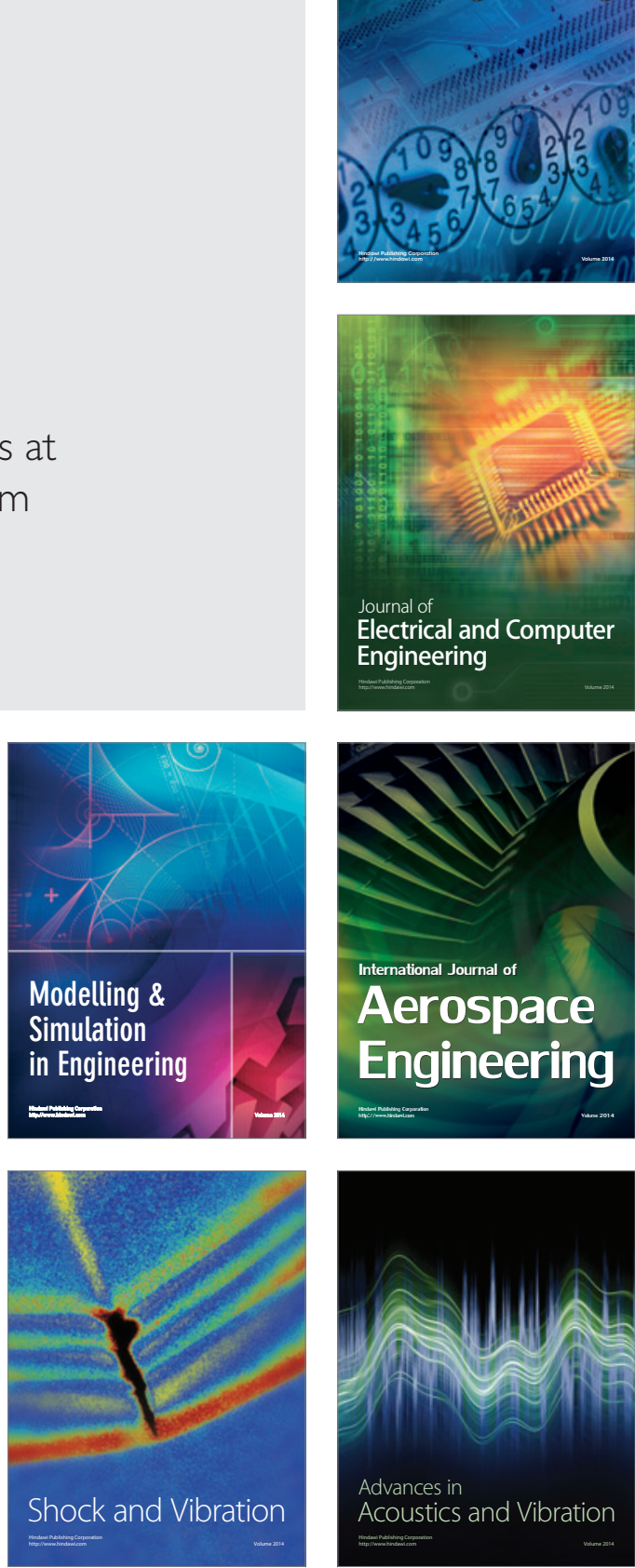